\title{
A case of relapsing iritis with hypopyon: translation of the original account of Benediktos Adamantiades
}

\begin{abstract}
Benediktos Adamantiades presented an account of a 20-year-old male with oral and genital aphthosis, pyoderma and arthritis at a meeting of the Medical Association of Athens in November 1930. His case subsequently published in Greek, and then in French in 1931, and is recognized through the dual eponym 'Adamantiades-Behçet disease' by many current investigators. Adamantiades subsequently added other observations to the syndrome, and developed the first classification and diagnostic criteria for it. The translation of his work from French to English is offered to provide accessibility to a wider audience interested in the history of what is commonly called Behçet disease.
\end{abstract}

First draft submitted: 12 April 2016; Accepted for publication 27 April 2016; Published online 30 June 2016

Keywords: Adamantiades $\bullet$ Behçet disease $\bullet$ history of medicine $\bullet$ vasculitis

The description of patients with clinical features that we now refer to as Behçet's disease can be traced through medical writings from Hippocrates to authors of modern times [1-7]. Significant contributions to modern understanding of what is known as Behçet's disease were made by Benediktos Adamantiades. Adamantiades wrote his initial significant contribution in Greek in 1930, followed by his contribution in French in 1931 [8,9]. The work by Hulusi Behçet was published in German in 1937; a translation of his work into English was published in 2013 [10]. The observation of Adamantiades is a testament to his powers of observation and scientific curiosity, and was important in the recognition of this group of symptoms and findings as a unique disorder. A translation of his account from French into English is provided to make his observations accessible to a wider audience.

Benediktos Adamantiades (Figure 1) was born in Prousa, Asia Minor, in 1875. He received his medical degree from the University of Athens, and then studied ophthalmol- ogy in Paris at Hotel Dieu and Quinze Vingts. In his practice in Athens, he was a tireless investigator, completing over 150 articles, making several premier observations [5].

Adamantiades presented 'On a case of relapsing iritis with hypopyon' at the meeting of the Medical Association of Athens in November 1930. This case was published that year in the Annals of the Medical Society of Athens and in 1931 in Annales d'Oculistique [8,9]. Adamantiades' work continues to be recognized through the faithful use of the dual eponym 'AdamantiadesBehçet disease' by many current investigators. In addition to his initial observations, Adamantiades is credited with the recognition of retinal and peripheral thrombophlebitis as part of this disorder [11]. He presented the first classification of the disease and diagnostic criteria; he recognized the poorer prognosis in males [5].

With this translation, I have attempted to remain true to the word and intent of the author, and have attempted to retain the original print style, punctuation and word
Eric L Matteson

Division of Rheumatology, Department of Internal Medicine \& Division of Epidemiology, Department of Health Sciences Research, Mayo Clinic \& Mayo Graduate School of Medicine, 200 1st St SW, Rochester, MN 55905, USA matteson.eric@mayo.edu

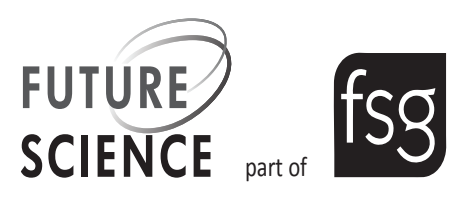




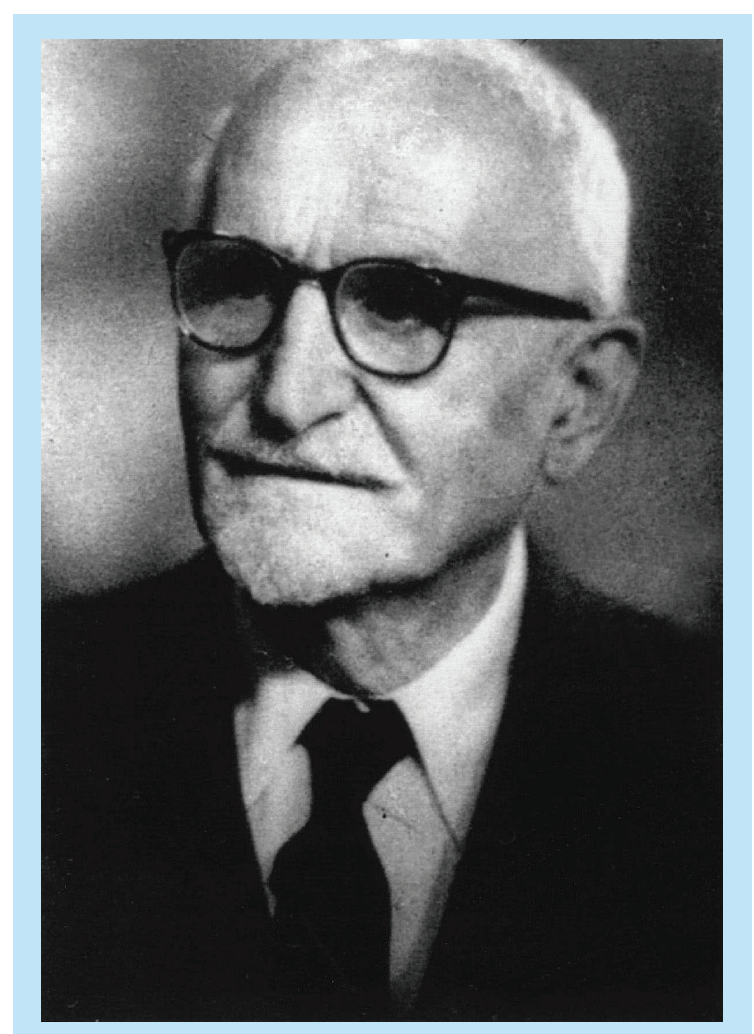

Figure 1. Benediktos Adamantiades.

usage as much as possible; some minor grammatical changes have been made to improve readability, and occasionally clarification of the original text has been provided in brackets.

\section{On a case of relapsing iritis with hypopyon} By B Adamantiades (Athens)

Cases of iritis with relapsing hypopyon are not encountered very frequently. It is for this reason that we wanted to report a case of this curious illness, which we have followed for 3 years, even more so because we could not find a detailed description in the French literature which could aid us in the understanding of its cause.

Observation - Gr. H., age 20 years, was admitted to the Athens Refugee Hospital on 28 June 1928.

His father died as a prisoner of war. His mother died of pulmonary tuberculosis. Two of his brothers died at a young age.

He had never had either gonorrhea or syphilis. At the age of 16, he contracted malaria. During the years 1925 and 1926, he had an ailing left leg. This was swollen and at the same time, some type of ulcerations appeared on its lower third. All of this was diagnosed by several physicians whom he had consulted as phlebitis, by some of the others as a manifestation of syphilis (despite his negative Wassermann) and by others as a simple pyoderma cured autovaccination.
In 1927, his eye was affected for the first time. At the dispensary of Kessariani, where we took him, we, at that time, had diagnosed an iritis with hypopyon. Examination of his blood at that time revealed a positive Wassermann. It was the only time his Wassermann was positive.

Since then, he has had unceasing new attacks of iritis in the one or in the other eye, always with hypopyon. Sometimes a cold preceded or accompanied the attack. At the same time, he began to have ulcerations of the scrotum, which reappeared after their rapid disappearance. Antisyphilitic therapy to which the patient was subjected had no influence on the course of the lesions. He does not suffer from tooth pain. He did not have erythema nodosum. For the past 2 months, he has not been able to see at all with his o.s. (left eye), and for the past 2 days, his o.d. (right eye) also began to be affected anew.

Current Status - o.d. hyperemic conjunctivitis. Intense ciliary injection. Some rare precipitates in the membrane of Descemet. Iris, sluggish, superficial posterior synechiae. Hypopyon of $1.5 \mathrm{~mm}$ height. Fundus, nonreflective. Intense periorbital pain. Visual acuity $=$ light perception.

o.s. atrophic eyeball. The anterior chamber no longer exists. Irregular pupillary margin of the iris. The papilla has a color reminiscent of that of an amaurotic cat. Very intense periorbital pains. Eyeball very sensitive to pressure in the ciliary region.

The general medical examination revealed no particular abnormalities. In the radiographs, we found a swelling of the hilar nodes of the left lung. Repeated testing of his blood, even following relapses, always resulted in a negative Wassermann.

We note that for a long time, the patient has been receiving antisyphilitic treatment. We are, incidentally, continuing it in combination with an appropriate local treatment.

A few days' stay in the hospital is sufficient that the hypopyon in his right eye disappeared. At the same time, the pupil is dilated, and the synechiae have begun to tear. A little later, the fundus of the eye has cleared up, and one could see a pale pupil with narrowed vessels despite an already affected vitreous.

In 2 months, all of the synechiae have torn, leaving behind crystalloid of the pigmentary deposits. The visual acuity $=0.05$.

In this period, we proceed with enucleation of his left eye, which has become very painful.

But on 24 August 1928, the hypopyon again appeared with a much more moderate intensity, only to disappear after a few days. The fundus of the eye cleared up again to the point that at the beginning of December 1928, the patient had a visual acuity $=0.1$. 
To the antisyphilitic treatment, we have pursued since his hospital admission, the last 2 months we have been adding injections of tuberculin. However, with the reappearance of his hypopyon, on 29 December 1928, we have, for some time, been trying protein therapy, which we quickly abandoned because of the intense reaction it provokes.

The hypopyon disappeared and reappeared on 11 February 1929 and after resolution, reappeared on 19 February.

Each relapse could only increase the amblyopia of the patient.

We are also noting here that since his admission to the hospital, he had, at times, an eruption of some ulcerations on his scrotum, which quickly scarred, only to reappear close to the scars they had left.

On 5 March 1929, the patient leaves the hospital with superficial posterior synechiae, with a pupil in semi-dilatation and a rather affected vitreous. Pressure by Schiolz, 11/7.5. Visual acuity = counts fingers at 1 meter.

We learned that the patient has pursued an antisyphilitic treatment since his discharge. It is during this treatment that the hypopyon reappeared and that the patient again was admitted to the hospital on 27 June 1929. At that point, we had him undergo a general examination, to the extent that the means of our hospital permits. Radiography of the lungs again shows hilar adenopathy. The otorhinolaryngologic examination reveals the existence of a tonsil with a chronic rhinopharyngitis (on the surface, his tonsil staphylococci were found a few months later). His left knee as well as his left leg is edematous. On the outer surface of the lower part of that leg, one sees five to six pigmented scars of $8-10 \mathrm{~mm}$ in diameter. On the scrotum, one sees round ulcerations with prominent margins and a proper base simultaneously with scars from old ulcerations. On the one hand, they are characteristic of syphilitic exanthems, on the other hand, an eruption of herpes. The inguinal lymph nodes, those of the axillae, as well as the left epitrochlear are swollen. One also notes ulcerations in the mouth.

Locally, one notices anew the existence of an iritis with hypopyon with abundant synechia and a nonreflective fundus. Visual acuity = light perception.

Treatment with Bismuth. The hypopyon, as usually, disappears in a few days but reappears on 3 August and again is reabsorbed.

We take advantage of the quiescent state of his eye, and on 4 December 1929, we proceed with a large superior iridectomy. But the hypopyon appears a few days after the intervention (9 December), resorbs and reappears on 16 January 1930, and again on 20 February 1930.
We now begin a treatment with intramuscular injections of electrorgol and intravenous sodium salicylate, and finally we proceed with the formation of fixed abscess. All of this treatment has not had any influence on the course of this disease.

Culture of the hypopyon pus obtained by aspirating the anterior chamber was negative, and inoculation of a rabbit's eye provoked an aseptic purulent inflammation with a negative examination as well (Pasteur Institute of Athens, Greece).

We should also make a note that during his second stay in the hospital, the patient began to have symptoms in both knees. A periodic hydarthosis affected first one of his knees, then the other, with almost no reactive inflammatory manifestations. It disappeared a few hours after it appeared only to reappear again. Radiographs revealed nothing particular in the joints. However, after 3 or 4 months, the hydarthrosis began to bother him and we were obliged to perform repeated aspirations. The aspirated fluid was at first serous, clear, but later became turbid. The culture was negative. Inoculation of the guinea pig also yielded no result.

In the meantime, the patient periodically had an eruption of oral aphthae and formation of a tonsillar abscess. In the same manner, the hypopyon appeared and disappeared on 19 March, 2 April, 15 April and 29 May 1930.

In June, culture of the surface of the scrotal ulcerations taken at several times was negative for Treponema but positive for Staphylococcus.

On 20 June, examination of the cerebral spinous fluid revealed albumin, $0.25,0.50$ glucose, very few lymphocytes and a negative Wasserman.

On 21 July, 28 July and 5 October, and 12 October, we have renewed appearances and disappearances of the hypopyon with almost no signs of irritation, (inflammatory).

On 3 October, the blood culture revealed white Staphylococcus. Autovaccination leads to fever and worsened general medical status (physical decline).

His current state (20 October 1930) is summarized as follows: cornea full of punctations localized in the anterior bed of stroma. Atrophic iris with a pigmented border measuring 1-2 mm. Pigment granules are deposited on the crystalloid. Despite difficulty visualizing the fundus, one sees an atrophic papilla. Vision $=$ doubtful light perception .

At the same time, his general health is bad. The patient has become pale, anemic and thin.

Before us, the clinical features of the disease which Gilbert has named ophthalmia lenta (12).

What characterizes the disease is hypopyon without many inflammatory (reactionary) phenomena and its rapid resolution and reappearance after some time. 
Each relapse leaves more or less permanent lesions, which sooner or later lead to blindness.

In our case, the blindness is due to atrophy of the optic nerve, which, according to Meller (13), itself was caused by the loss of cellular debris by the optic nerve sheaths.

As we have reiterated, the disease is accompanied by minimal reactionary (inflammatory) phenomena. A mild ciliary injection precedes the appearance of the hypopyon by several hours $(20-30 \mathrm{~h})$. Sometimes, this same ciliary injection resolves without formation of a hypopyon.

The variety of generally applied treatment during the long duration of the illness reveals our uncertainty regarding etiology. What could therefore be the cause of this iritis, at least in our case?

To find the etiologic agent of each iritis, one cannot but base it on two elements: on the form in which the iritis appears; on the findings which the general medical examination (based on) other coexistent manifestations could provide.

The form in which the iritis appears can only rarely contribute to ascertainment of the underlying cause. The appellation serous, fibrinous, among others, iritis, which one finds in the literature provides no hint of its cause. A syphilitic or tuberculous iritis can present as a nodular form or a serous form with abundant precipitates or a fibrinous form with marked synechiae, or a purulent form. It is very justified that Venneman (13) has proclaimed that the body reacts in the same manner to the most varied irritants. One can go even further and assert that neither the table (tableau schema) which gives us the Gullstrand lamp nor even the histologic examination can, in many cases, give us an element of certainty about the underlying etiology.

If we now return to our case of relapsing iritis with hypopyon, a brief overview of its history could more than anything else remind us of the uncertainty which prevails from the point of view of its etiology.

It was Reis who first describes the disease in 1906 (Kl. Mon. f. Aug.) as a metastatic iritis in connection with a nodular erythema.

In 1917, Köppe (Gr. Arch.) describes another case of relapsing bilateral iritis with hypopyon.

In 1920, Gilbert (Arch. F. A) in describing another case agrees with Reis with respect to the dependence of the erythema.

In 1922, Stahli (Kl. M. f. A) is of the opinion that the disease is of a tuberculous nature.

Weve (1923) is a proponent of a staphylococcal causation.

Urbanek (Zeit f. Aug. 1029) describes two cases which he attributes to tuberculosis.
In 1930, Gilbert in his article about the iritocyclitides (K Handbuch der Ophtalmologie) is of the opinion that this iritis is due to chronic infections or aiguës or suppurations (erythema nodosa, pyoderma, furunculosis, arthritis, among others), and he adds that in one of these cases, he could culture staphylococcus from the blood as well as from the abscess (locus of pus; foyer primitif du pus). We, therefore, appreciate the uncertainty regarding the etiology of the disease.

The culture and inoculation of the pus from the hypopyon which could perhaps have given us a greater degree of certainty was negative in our case, as well as those of Urbanek. Therefore, the way the disease presents itself cannot enlighten us from the point of view of its etiology.

Let us now examine whether the general state of the patient could help us in our task.

The study of his general condition as we have described it can only place into question one of three etiologic agents: tuberculosis, syphilis and Staphylococal infection.

From the point of view of tuberculosis, the patient presents a hereditary predisposition and swelling of the hilar lymph nodes. The role, which these past years has been attributed to tuberculosis as an etiologic factor of the diseases of the eyes and above all diseases of the uvea is well known. It is I. Von Michel who first stated that tuberculosis of the iris (a cardinal manifestation of systemic infection) is quite frequent $(50 \%$ of cases of iritis). Meller (13) also stated that among 500 eye patients, more than half are of a tuberculous nature. Gilbert (12) considers $45 \%$ of 500 cases of iridocyclitis as tuberculous. Let us note that one does not find iritis among serious and diagnosed forms of tuberculosis, but rather in the mild forms, that or after a thorough search, one can rarely diagnose tuberculosis.

Can we then accept tuberculosis as the causative agent in our case? Certainly, one cannot exclude it, despite the failure of the antituberculous treatment which we had attempted.

But let us now examine the two other agents. From the point of view of syphilis, we have the assurances of our dermatologists who declared that the patient's diverse cutaneous manifestations were syphilitic (secondary, tertiary or hereditary phenomena) and explain that the resistance of all of these manifestations to antisyphilitic treatment is due to a particular resistance of the patient to three specific medications. However, although we are sure of the syphilitic nature of all of these manifestations, we could not, therefore, conclude that some manifestation superimposed on a syphilitic lesion must with certainty be a syphilitic nature. Let us also admit that the resistance of the patient to treatment as well as the negative result of the sero- 
logic examination impresses us unfavorably as to the syphilitic etiology.

But, let us go to the third agent. Let us say at the outset that what was remarkable about our patient from a clinical point of view is that the scrotal lesions, the hydrarthroses and the appearance of the iritis were part of the same process. All of these manifestations appeared, persisted some days and disappeared, only to reappear after a variable interval without being influenced by any type of treatment.

If we combine these clinical phenomena with the observation of staphylococcus in part in the scrotal ulcerations in part on the pharynx and in the blood, we are not far from the hypothesis of a focal infection. In the English speaking countries, the focal infection forms a very important chapter in pathology, even if a little nebulous. We know that the theory of focal infections is composed of many generalized or localized infectious manifestations that depend on the existence somewhere in the body, sometimes remote, of one or more, very limited, deep, circumscribed microbial foci, which only manifest themselves in insignificant clinical signs and which demonstrate no relationship between their severity and that of the disease which they determine. They are seated, for example, in the tonsils, the teeth, among others.

Therefore, it is probable that the recurrent hypopyon is due to a transient but recurrent bacteremia,

\section{References}

1 Blüthe L. Zur Kenntnis des recidiverenden hypopyons. In: Inaugural Dissertation. Heidelberg, Germany, (1908).

2 Planner H, Remenovsky F. Beitrage zur Kenntnis der Ulcerationen am äusseren weiblichen Genitale. Arch. Dermatol. Syphilol. 140, 162-188 (1922).

3 Shigeta T. Recurrent iritis with hypopyon and its pathological findings. Acta Soc. Ophthalmol. Jpn. 28, 516 (1924).

4 Matteson EL. Notes on the history of eponymic idiopathic vasculitis: the diseases of Henoch and Schonlein, Wegener, Churg and Strauss, Horton, Takayasu, Behçet, and Kawasaki. Arthritis Care Res. 13(4), 237-244 (2000).

5 Whitwell GPB. Recurrent buccal and vulval ulcers with associated embolic phenomena in skin and eye. $\mathrm{Br}$. J. Dermatol. 46, 414-419 (1934).

6 Matteson EL. On relapsing, aphthous ulcers of the mouth, eye and genitalia caused by a virus. Translation of the original article by Hulusi Behçet. Clin. Exp. Rheumatol. 28(Suppl. 60), S2-S5 (2010). which metastasizes (sallies reaches Ausschwärmen to the level of) to the iris.

We, therefore, believe that it is the Staphylococcus that periodically entering the circulation, as the blood culture has demonstrated, and which from time to time localizes in the iris and provokes this recurrent iritis with hypopyon, at least in our case $[12,13]$.

\section{Acknowledgments}

My sincere thanks to Herta Matteson and Shreyasee Amin for their assistance in the preparation of this translation.

\section{Financial \& competing interests disclosure}

The author has no relevant affiliations or financial involvement with any organization or entity with a financial interest in or financial conflict with the subject matter or materials discussed in the manuscript. This includes employment, consultancies, honoraria, stock ownership or options, expert testimony, grants or patents received or pending, or royalties.

No writing assistance was utilized in the production of this manuscript.

\section{Open Access}

This work is licensed under the Creative Commons Attribution 4.0 License. To view a copy of this license, visit http://creativecommons.org/licenses/by/4.0/

7 Adamantiades B. La thrombophlebite comme quatrieme symptome de l'iritis recidivante a hypopyon. Ann. Oculist 179(3), 143-148 (1946).

8 Behçet H. Ueber rezidivierende aphthoese, durch ein Virus verursachte Geschwuere am Mund, am Auge und an den Genitalien. Derm. Wochenschr. 36, 1152-1157 (1937).

9 Zouboulis CC, Kietel W. The history of AdamantiadesBehçet's disease. J. Invest. Dermatol. 119(1), 201-205 (2002).

10 Adamantiades B. A case of relapsing iritis with hypopyon. In: Archia Iatrikis Etairias. Athens, Greece, 586-593 (1930).

11 Adamantiades B. Sur un cas d'iritis a hypopion recidivant. Ann. Ocul. 168, 271-278 (1931).

12 Gilbert W. Die Erkrankungen der Uvea. Handbuch der Ophthalmologie. Schieck F, Bruckner A (Eds). Springer, Berlin, Germany, 30 (1930).

13 Meller J. Chronische iridocyclites und neuritis retrobulbares Gr. C/B. 105. Enc. D’Opht.fr., t. VI.6. Masson \& Cie, Paris, France, 1924. 